\title{
Transportation mode and timing of spinal cord decompression and stabilization in patients with traumatic spinal cord injury in Iran
}

\author{
Mahsa Ghajarzadeh ${ }^{1} \cdot$ Hooshang Saberi ${ }^{1,2}$
}

Received: 14 January 2018 / Revised: 5 July 2018 / Accepted: 23 July 2018 / Published online: 10 September 2018

(c) International Spinal Cord Society 2018

\begin{abstract}
Study design Cross-sectional retrospective study.

Objective To describe the transportation mode to hospital and timing of spinal cord decompression and stabilization (D\&S), length of hospital stay, frequency of pressure injuries, and sepsis during hospitalization.

Setting Brain and Spinal Injury Research Center, Tehran, Iran.

Methods Eight hundred and thirty patients with traumatic spinal cord injury (TSCI) were enrolled. Mode of transportation and length of time to reach the first hospital, length of hospital stay (LOS), and the time span between hospital arrival and decompression and stabilization (D\&S) were recorded.

Results Fifty-nine percent of the enrolled individuals were transported to the first hospital by ambulance, while $41 \%$ were transferred by vehicles without medical equipment and personnel. Median length of time to reach the first hospital was $1 \mathrm{~h}$ for both ambulance and non-equipped car groups, with no statistically significant difference $(p=0.1)$. Median LOS, frequencies of pressure injuries, and sepsis based on the injury levels were not significantly different between two transportation modalities. One hundred and seventy-seven individuals had early surgery, and 254 had late surgery. Median LOS was 13 days in the early surgery group and 20 days in the late surgery group $(p=0.002)$. Frequencies of pressure injuries and sepsis were not significantly different between the late and early surgery groups for various injury levels.

Conclusion About 59\% of our patients had been transported to a hospital by non-medical personnel. Those with late surgery had significantly longer LOS. Improving TSCI patients' transportation method and early surgical interventions, if possible, may be considered.
\end{abstract}

\section{Introduction}

Traumatic spinal cord injury (TSCI) is one of the important causes of long-term disability and health-care cost in lowand middle-income countries [1]. Its heavy burden on affected individuals, their families, and society is due to the high costs of the acute phase treatments, long rehabilitation periods, as well as loss of productivity and the emotional impact on their lives [2]. The final functional outcome of the patients with TSCI depends on the injury level and severity,

Hooshang Saberi

hgsaberi@yahoo.com

1 Brain and Spinal Cord Injury Research Center, Tehran University of Medical Sciences, Tehran, Iran

2 Department of Neurosurgery, Tehran University of Medical Sciences, Tehran, Iran pre-hospital and triage care, and the timing of spinal cord decompression and stabilization (D\&S) [3]. One of the challenging issues in the management of TSCI has been the timing of $\mathrm{D} \& \mathrm{~S}$ in the hope of preservation and/or restoration of damaged neural tissue for better neurological functioning [3]. The incidence of TSCI has increased in recent years, with an annual incidence of about 10.4-83 cases per million in various countries worldwide [4]. One of the important issues in patients with TSCI is pre-hospital care and management. The unstable spine will increase the severity of a neurological injury, so immobilization before reaching the hospital is a topic of concern [5], preventing secondary injury induced by inappropriate transfer modalities or spinal immobilization [6]. Previous studies have shown that nearly one-fourth of spinal injuries may worsen after the primary injury before hospital arrival [7]. Therefore, the pre-hospital mode of transportation should be considered carefully.

The World Health Organization (WHO) recommends more research on prevention of secondary injuries, especially 
in low- and middle-income countries [8]. Similarly, the time span from admission to spine stabilization surgery, if indicated, may influence LOS and frequency of pressure injuries and sepsis during hospitalization, as well as the final outcome [9]. As far as we know, information regarding transportation mode and timing of surgery is limited for individuals with TSCI, and such knowledge may be of great importance for health policy makers in low- and middle-income countries. Therefore, we designed this study to describe the pattern of hospital transportation, timing of surgery, LOS, and the frequency of pressure injuries and sepsis during hospitalization in a cohort of individuals with TSCI.

\section{Methods}

This retrospective study was conducted at the Brain and Spinal Injury Research Center, Tehran, Iran between March 2013 and May 2017. Participants filled informed consent forms before enrollment in the study. The ethical committee of TUMS (Tehran University of Medical Sciences) approved the study protocol (ID:25661). Inclusion criteria were: established TSCI (for at least 1 year), valid medical records regarding transportation mode to the first hospital, the timing of surgery, and occurrence of a pressure injury (grade more than II, size $>5 \mathrm{~cm}$ ), and sepsis (systemic inflammatory response syndrome plus three consequent positive blood cultures) during the hospital stay. The participants were categorized into cervical $(N=73)$, thoracic $(N=627)$, and lumbar $(N=130)$ groups. Data regarding etiology of injury, transportation mode to the first hospital, level and severity of injury (ASIA impairment scale (AIS)), the time span between injury and the first hospital arrival, overall length of stay (LOS), and the time span between the first hospital arrival and surgery were extracted from the medical records of the included participants. Statistical analysis for subgroups, based on the level of the injury, was performed considering the mode of transportation, and timing of D\&S as independent variables. LOS, significant pressure injuries, and sepsis were considered as outcomes. The median LOS in the different subgroups was compared using the Wilcoxon-Mann-Whitney test, while pressure injury and sepsis frequencies were compared using the $\chi^{2}$ test. Data analysis was conducted using SPSS software, version 22 (IBM, Chicago, IL, USA). A $p$ value $<0.05$ was considered to be statistically significant.

\section{Results}

Of the 1024 individuals eligible for the study, 112 were unwilling to participate. An additional 82 were excluded because of inconclusive information about transportation mode, timing of D\&S, and complications during the hospitalization period. Therefore, 830 individuals were enrolled. The mean $( \pm$ SD) age of participants was $36.9( \pm 11.5)$ years, and the male to female ratio was 4.3/1 (Table 1). Four hundred and thirty-one individuals had D\&S and the remainder underwent conservative management. Overall, the median time to reach the first hospital was $1 \mathrm{~h}$ (interquartile range (IQR): 1-2). The median time span between hospital arrival and surgery (in 431 individuals) was 3 days (IQR: 1-7), and the median overall hospital LOS was 15 days (IQR: 8-30). Fifty-nine percent of our participants were transferred to the first hospital by emergency equipped ambulances and $41 \%$ were transported with unequipped cars (without any facilities such as backboard, collars, and paramedics). The median time to reach the first hospital was $1 \mathrm{~h}$ (IQR: 1-2) for both ambulance and unequipped car groups (IQR: $1-2)(p=0.1)$. The median LOS was 17 days (IQR: 10-30) for those with cervical TSCI, 15 days (IQR: 8-30) for those with thoracic TSCI, and 15 days (IQR: $7-27)$ for those with lumbar TSCI $(p=0.04)$. The median LOS in the C1-C4 cervical injury group was 30 days (IQR: 14-60), while it was 15 days (IQR: 9-30) in the C5-C7 group $(p=0.03)$. The level of injury was significantly different between the two transportation modes. However,

Table 1 Baseline characteristics of patients

\begin{tabular}{ll}
\hline Variables & Frequency/mean $( \pm \mathrm{SD})$ \\
\hline Age at the time of the study (years) (mean \pm & $36.9 \pm 11.5$ \\
SD) & \\
Gender $(\boldsymbol{N} \%)$ & $674(81 \%)$ \\
Male & $156(19 \%)$ \\
Female & \\
Level of injury $(\boldsymbol{N} \%)$ & $13(1 \%)$ \\
C1-C4 & $60(7 \%)$ \\
C5-C7 & $162(20 \%)$ \\
T1-T6 & $465(56.0 \%)$ \\
T7-T12 & $130(16 \%)$ \\
L1-L5 & $537(65.0 \%)$ \\
Neurological status after acute treatments & AIS) $(\boldsymbol{N \%})$ \\
A & $126(15.0 \%)$ \\
B & $131(16.0 \%)$ \\
C & $36(4.0 \%)$ \\
D & \\
Etiology (n\%) & $512(62 \%)$ \\
Vehicle accidents & $238(29 \%)$ \\
Falling & $80(9 \%)$ \\
Others & \\
Transfer to primary center $(\boldsymbol{N} \%)$ & $391(59 \%)$ \\
Ambulance with paramedics & \\
Non-equipped cars & \\
\hline
\end{tabular}


AIS, LOS, and complication rate were not significantly different between the transportation modes (Table 2). The median LOS as well as frequencies of pressure injuries and sepsis were not different between the two transportation modes for different levels of spinal injury (Table 3 ). One hundred and seventy-seven had D\&S during the first $48 \mathrm{~h}$ after hospital arrival, and 254 underwent surgery after $48 \mathrm{~h}$. The median LOS was 13 days (IQR: 7-21) in the early surgery group, and 21 days (IQR: 14-21) in the late surgery group $(p=0.002)$. We performed subgroup analysis in the 431 operated cases according to the timing of D\&S in different neurological levels separately. Our results in the cervical group showed a median LOS of 14 days (IQR: 7-30) in early D\&S, and 25 days (IQR: 10-36) in the late group $(p=0.01)$. In the thoracic subgroup, the median LOS

Table 2 Comparison of different factors in cases who were transported by ambulance or unequipped cars

\begin{tabular}{|c|c|c|c|}
\hline & Ambulance $N=491$ & $\begin{array}{l}\text { Own } \operatorname{car} N= \\
339\end{array}$ & $p$ value \\
\hline \multicolumn{4}{|c|}{ Level of the injury $(N \%)$} \\
\hline Cervical & $43(59 \%)$ & $30(41 \%)$ & \multirow[t]{3}{*}{0.008} \\
\hline Thoracic & $387(62 \%)$ & $240(38 \%)$ & \\
\hline Lumbar & $61(47 \%)$ & $69(53 \%)$ & \\
\hline \multicolumn{4}{|l|}{ ASIA $(N \%)$} \\
\hline A & $315(59 \%)$ & $222(41 \%)$ & \multirow[t]{4}{*}{0.9} \\
\hline B & $74(59 \%)$ & $52(41 \%)$ & \\
\hline $\mathrm{C}$ & $81(62 \%)$ & $50(38 \%)$ & \\
\hline $\mathrm{D}$ & $21(58 \%)$ & $15(42 \%)$ & \\
\hline \multicolumn{4}{|c|}{ Complications $(N \%)$} \\
\hline Pressure ulcers & $125(57 \%)$ & $96(43 \%)$ & 0.3 \\
\hline Sepsis & $6(1.2 \%)$ & $1(0.2 \%)$ & 0.1 \\
\hline LOS $($ mean $\pm \mathrm{SD})$ & $25.2 \pm 38.1$ & $26.6 \pm 49$ & 0.6 \\
\hline
\end{tabular}

LOS length of stay was 11 days (IQR: 7-30) in the early D\&S group, and 20 days (IQR: $11-30)$ in the late surgery group $(p=0.03)$. In the lumbar subgroup, the median LOS was 14 days (IQR: 6-20) in the early group and 18 days (IQR: 10.5-30) in the late group $(p=0.01)$. Nevertheless, the frequency of significant pressure injuries and sepsis was not significantly different in the late and early D\&S groups for different injury levels (Table 4).

\section{Discussion}

Although there have been many attempts to reduce the incidence and prevalence of TSCI worldwide, it is everincreasing [10]. Individuals who are suspected to have TSCI at the scene should receive great care before hospital arrival [6]. There are wide variations regarding pre-hospital care and transportation modes in different communities. However, there is evidence that neurological deterioration may occur in 10-26\% of patients with TSCI during pre-hospital transport [11, 12].

Regarding mode of transport in our study, overall, 59\% of our participants had been transported to the first hospital by ambulance. In a previous study from the USA, medical records of 61 patients with acute TSCI were reviewed. Nearly $41 \%$ had been transported by ground ambulance, $54 \%$ by helicopter, and $5 \%$ by fixed-wing aircraft, all equipped with paramedics [13]. In a study conducted by Flabouris from Australia, $93 \%$ of patients suspected to have TSCI had been transported by helicopter, $3.5 \%$ by aircraft, and $3.5 \%$ by road vehicles [12]. In our study, $41 \%$ of patients were transported to the first hospital by unequipped cars, which shows a huge gap between accepted standards and the current situation for emergency transportation in Iran. The high rate of unequipped road vehicle transportation in Iran, as a low income country, may be approached as
Table 3 Comparison of length of stay, pressure injuries, and sepsis in patients who were transferred by ambulance or unequipped cars based on different injury levels

\begin{tabular}{|c|c|c|c|}
\hline & Ambulance transferred & Non-equipped car & $p$ value \\
\hline Cervical group & $N=43$ & $N=\mathbf{3 0}$ & \\
\hline LOS, median (IQR) & 18 (IQR: 18-40) & 16 (IQR: 8-30) & 0.2 \\
\hline Pressure ulcer $(N \%)$ & $8(18.6 \%)$ & $6(20 \%)$ & 0.8 \\
\hline Sepsis $(N \%)$ & $2(4.6 \%)$ & $1(3.3 \%)$ & 0.7 \\
\hline Thoracic group & $N=\mathbf{3 8 7}$ & $N=\mathbf{2 4 0}$ & \\
\hline LOS, median (IQR) & 15 (IQR: 7-30) & 15 (IQR: 8-30) & 0.4 \\
\hline Pressure ulcer $(N \%)$ & $100(25.8 \%)$ & $73(30.4 \%)$ & 0.2 \\
\hline Sepsis $(N \%)$ & $3(0.9 \%)$ & - & 0.1 \\
\hline Lumbar group & $N=61$ & $N=68$ & \\
\hline LOS, median (IQR) & 16 (IQR: 10-30) & 14 (IQR: 5-24) & 0.2 \\
\hline Pressure ulcer $(N \%)$ & $17(4.3 \%)$ & $17(7 \%)$ & 0.6 \\
\hline Sepsis $(N \%)$ & $1(1.6 \%)$ & 0 & 0.2 \\
\hline
\end{tabular}

$\operatorname{LOS}$ length of stay 
Table 4 Comparison of length of stay, pressure injuries, and sepsis in patients in early and late surgery groups based on different injury levels

\begin{tabular}{clll}
\hline & Early surgery group & Late surgery group & $p$ value \\
\hline Cervical spine injury & $\boldsymbol{N}=\mathbf{1 9}$ & $\boldsymbol{N}=\mathbf{4 1}$ & \\
LOS, median (IQR) & $14(\mathrm{IQR}: 7-30)$ & 25 (IQR: $10-36)$ & 0.01 \\
Pressure ulcer (N\%) & $2(10.5 \%)$ & $10(24.3 \%)$ & 0.2 \\
Sepsis $(N \%)$ & 0 & $2(4.8 \%)$ & 0.3 \\
Thoracic spine injury & $\boldsymbol{N}=\mathbf{1 2 0}$ & $\boldsymbol{N}=\mathbf{1 4 4}$ & \\
LOS, median (IQR) & $11(\mathrm{IQR}: 7-0)$ & $20(\mathrm{IQR}: 11-30)$ & 0.03 \\
Pressure ulcer $(N \%)$ & $34(28.3 \%)$ & $40(27.7 \%)$ & 0.9 \\
Sepsis $(N \%)$ & $1(0.8 \%)$ & $1(1.1 \%)$ & 0.3 \\
Lumbar spine injury & $\boldsymbol{N}=\mathbf{3 8}$ & $\boldsymbol{N}=\mathbf{6 9}$ & \\
LOS, median (IQR) & $14(\mathrm{IQR}: 6-20)$ & $18(\mathrm{IQR}: 10.5-30)$ & 0.01 \\
Pressure ulcer $(N \%)$ & $13(34.2 \%)$ & $18(26 \%)$ & 0.3 \\
Sepsis $(N \%)$ & $1(2.6 \%)$ & 0 & 0.1 \\
\hline LOS length of stay & & &
\end{tabular}

Pickett et al. [17] reported shorter hospital LOS for those in the lumbar subgroup in their study. Our results also show that median LOS was longer for individuals in the cervical group in comparison with other two injury levels. Longer LOS may be associated with further complications, cost, and need for medical care.

The optimal timing for stabilizing and fixation for spinal fractures has been controversial with no consensus [3]. Early removal of the bone, disc, and ligamentous fragments compressing the spinal cord may lead to inhibition of secondary neural damage. Some authors believe that longer compression time may be associated with less neurological recovery $[19,20]$. Some studies have reported benefits for early spinal fixation such as shorter duration of hospitalization and ICU stay, better neurological improvement, shorter ventilation period, and less incidence of hospitalacquired pneumonia $[9,21]$. Our study showed that the median LOS was significantly longer in the late surgery group for all injury levels, while the frequency of pressure injuries and sepsis was not significantly different between neurological level subgroups.

Early decompression in patients with SCI (during 8-10 h) may result in better neurological recovery [22-24]. In addition, early surgical interventions may reduce the hospitalization period [3], which protects patients from different complications such as pressure injuries and sepsis. Cengiz et al. [3] in Turkey enrolled 27 individuals with thoracolumbar spine injuries. Their group "one" patients were medically stable and underwent surgery during the first $8 \mathrm{~h}$, while group "two" underwent surgery between 3rd and 15th day post injury. They reported shorter LOS and fewer complications in group one. This was consistent with our results; in our study, based on injury levels, LOS was significantly longer in the late D\&S group than the early group, although the rates of complications were similar. Bliemel et al. [25] in Germany evaluated documents of 
8994 individuals with TSCI. They found early surgery $(<72$ $\mathrm{h}$ post injury) resulted in shorter hospital LOS as well as fewer complications post-operatively. Fehlings et al. [26] followed up 222 individuals with acute cervical spine injury for 6 months and found that in nearly $24.2 \%$ of those who had undergone early surgery ( $<24 \mathrm{~h}$ post injury) showed at least one postoperative complication, as compared to the late surgery group ( $>24 \mathrm{~h}, 30.5 \%$ ), but the difference was not significant. In the study by Bliemel et al. [25], the rate of sepsis was significantly lower in the early surgery group. In a large study of 4354 individuals with SCI with either thoracic or lumbar injuries, early surgery reduced the ventilation period, ICU admission length, and overall hospital stay in both groups [25]. This was consistent with our study, where LOS was significantly shorter in the early surgery group for all injury levels. However, this finding was in contrast to Konieczny et al. [27]. In their study, hospitalization period was not significantly different between the early and late surgery groups ( $<72 \mathrm{~h}$ vs $>72 \mathrm{~h}$ post injury) [27], although the cutoff points were different. Only one previous study chose the cutoff point of $48 \mathrm{~h}$ for early or late surgery subgroups, and reported better neurological improvement in the early group [28].

Regarding the length of time to reach the first hospital, Leal-Filho [29] assessed 386 patients with TSCI in the acute phase in a public institute in Brazil. Most (47\%) had been admitted to the hospital $4-12 \mathrm{~h}$ after trauma, while the median duration to reach the first hospital was $1 \mathrm{~h}$ in our study (IQR: 1-2) for both the ambulance and unequipped car groups. Consequently, the important issue is not the length of time of hospital transport but the quality of the patient transport.

The pivotal point of this study was to consider the transportation mode and time span of the surgical intervention for TSCI and to compare LOS as well as complications in these groups. The study had limitations; we did not have baseline neurological data (at the scene) to objectively determine the rate of neurological deterioration during hospital transport. Furthermore, about one-fifth of the enrolled individuals were lost to follow-up. Future studies registering the first AIS (at the scene) for those with $\mathrm{SCI}$ is recommended to compare neurological deterioration. Also, multicentre studies with close follow-up planning and control of confounders in Iranian patients with TSCI are recommended.

As recommended by WHO, more studies should be done in low- and middle-income countries to clarify different aspects of injuries and associated problems to prepare information for health-care providers [8]. Supplying more equipped cars (ambulances) and helicopters or aircraft to transfer Iranian patients with TSCI is a major concern. On the other hand, early D\&S may be recommended in selected TSCI cases.

\section{Conclusion}

Sixty percent of our patients had been transported to the hospital by unequipped cars. Patients with late surgery had significantly longer LOS. Steps need to be taken to improve TSCI transportation mode and access to early surgery in Iran.

Funding This study was supported by Tehran University of Medical Sciences.

Author contributions M.G. contributed to study design, data gathering, data analysis, and manuscript writing. H.S. contributed to study conception, data gathering, data interpretation, and article writing.

\section{Compliance with ethical standards}

Conflict of interest The authors declare that they have no conflict of interest.

\section{References}

1. Selassie A, Cao Y, Saunders LL. Epidemiology of traumatic spinal cord injury among persons older than 21 years: a population-based study in South Carolina, 1998-2012. Top Spinal Cord Inj Rehabil. 2015;21:333-44.

2. Behrman AL, Trimble SA. Outcomes of spinal cord injuries in young children. Dev Med Child Neurol. 2012;54:1078

3. Cengiz ŞL, Kalkan E, Bayir A, Ilik K, Basefer A. Timing of thoracolomber spine stabilization in trauma patients; impact on neurological outcome and clinical course. A real prospective (rct) randomized controlled study. Arch Orthop Trauma Surg. 2008;128:959-66.

4. Cripps R, Lee B, Wing P, Weerts E, Mackay J, Brown D. A global map for traumatic spinal cord injury epidemiology: towards a living data repository for injury prevention. Spinal Cord. 2011;49:493-501.

5. Kwan I, Bunn F, Roberts IG. Spinal immobilisation for trauma patients. Cochrane Database Syst Rev. 2001;CD002803.

6. Ahn H, Singh J, Nathens A, MacDonald RD, Travers A, Tallon J, et al. Pre-hospital care management of a potential spinal cord injured patient: a systematic review of the literature and evidencebased guidelines. J Neurotrauma. 2011;28:1341-61.

7. Theodore N1, Hadley MN, Aarabi B, Dhall SS, Gelb DE, Hurlbert RJ, et al. Prehospital cervical spinal immobilization after trauma. Neurosurgery. 2013;72 Suppl 2:22-34. https://doi.org/10.1227/ NEU.0b013e318276edb1.

8. World Health Organization. Global status report on road safety: time for action. Geneva: World Health Organization; 2009.

9. McLain RF, Benson DR. Urgent surgical stabilization of spinal fractures in polytrauma patients. Spine. 1999;24:1646.

10. Yang R, Guo L, Huang L, Wang P, Tang Y, Ye J, et al. Epidemiological characteristics of traumatic spinal cord injury in Guangdong, China. Spine. 2017;42:E555-61.

11. Toscano J. Prevention of neurological deterioration before admission to a spinal cord injury unit. Spinal Cord. 1988;26:143-50.

12. Flabouris A. Clinical features, patterns of referral and out of hospital transport events for patients with suspected isolated spinal injury. Injury. 2001;32:569-75. 
13. Burney RE, Waggoner R, Maynard FM. Stabilization of spinal injury for early transfer. J Trauma. 1989;29:1497-9.

14. Hassani SA, Moharari RS, Sarvar M, Nejati A, Khashayar P. Helicopter emergency medical service inTehran, Iran: a descriptive study. Air Med J. 2012;31:294-7.

15. Perry SD, McLellan B, Mcllroy WE, Maki BE, Schwartz M, Fernie GR. The efficacy of head immobilization techniques during simulated vehicle motion. Spine. 1999;24:1839.

16. Domeier RM, Frederiksen SM, Welch K. Prospective performance assessment of an out-of-hospital protocol for selective spine immobilization using clinical spine clearance criteria. Ann Emerg Med. 2005;46:123-31.

17. Pickett GE, Campos-Benitez M, Keller JL, Duggal N. Epidemiology of traumatic spinal cord injury in Canada. Spine. 2006;31:799-805.

18. Güzelküçük Ü, Kesikburun S, Demir Y, Aras B, Özyörük E, Yılmaz B, et al. Demographic and clinical characteristics of patients with traumatic cervical spinal cord injury: a Turkish hospital-based study. Spinal Cord. 2015;53:441-5.

19. Tator $\mathrm{CH}$. Review of treatment trials in humanspinal cord injury: issues, difficulties, and recommendations. Neurosurgery. 2006;59:957-87.

20. Delamarter RB, Sherman J, Carr JB. Pathophysiology of spinal cord injury. Recovery after immediate and delayed decompression. J Bone Joint Surg Am. 1995;77:1042-9.

21. Frangen TM, Ruppert S, Muhr G, Schinkel C. Respiratory failure in thoracic spine injuries. Does the timing of dorsal stabilization have any effect on the clinical course in multiply injured patients? Orthopade. 2007;36:365-71.

22. Aebi M, Mohler J, Zäch G, Morscher E. Indication, surgical technique, and results of 100 surgically-treated fractures and fracture-dislocations of the cervical spine. Clin Orthop Relat Res. 1986;203:244-57.

23. Burke DC, Berryman D. The place of closed manipulation in the management of flexion-rotation dislocations of the cervical spine. J Bone Joint Surg Br. 1971;53:165-82.

24. Wiberg J, Hauge H. Neurological outcome after surgery for thoracic and lumbar spine injuries. Acta Neurochir. 1988;91:106-12.

25. Bliemel C, Lefering R, Buecking B, Frink M, Struewer J, Krueger A, et al. Early or delayed stabilization in severely injured patients with spinal fractures? Current surgical objectivity according to the Trauma Registry of DGU: treatment of spine injuries in polytrauma patients. J Trauma Acute Care Surg. 2014;76:366-73.

26. Fehlings MG, Vaccaro A, Wilson JR, Singh A, W Cadotte D, Harrop JS, et al. Early versus delayed decompression for traumatic cervical spinal cord injury: results of the Surgical Timing in Acute Spinal Cord Injury Study (STASCIS). PLoS ONE. 2012;7:23

27. Konieczny MR, Strüwer J, Jettkant B, Schinkel C, Kälicke T, Muhr G, et al. Early versus late surgery of thoracic spine fractures in multiple injured patients: is early stabilization always recommendable? Spine J. 2015;15:1713-8.

28. Clohisy JC, Akbarnia BA, Bucholz RD, Burkus JK, Backer RJ. Neurologic recovery associated with anterior decompression of spine fractures at the thoracolumbar junction (T12-L1). Spine. 1992;17:325-30.

29. Leal-Filho MB, Borges G, Almeida BR, Aguiar Ade A, Viera MA, Dantas Kda S, et al. Spinal cord injury: epidemiologycal study of 386 cases with emphasis on those patients admitted more than four hours after the trauma. Arq Neuropsiquiatr. 2008;66 (2B):365-8. 